\title{
Sistematização de experiência de acadêmicos de enfermagem no telemonitoramento em saúde durante a pandemia de coronavírus
}

\author{
Systematization of experience in health telemonitoring by nursing students during the coronavirus \\ pandemic
}

Sistematización de la experiencia en telemonitorización de la salud por parte de estudiantes de enfermería durante la pandemia de coronavirus

\author{
Luciene Oliveira da Cruz \\ ORCID: https://orcid.org/0000-0001-5634-0272 \\ Universidade Federal do Amazonas, Brasil \\ E-mail: Luciene.ocruz@gmail.com \\ Esron Soares Carvalho Rocha \\ ORCID: https://orcid.org/0000-0002-1011-6053 \\ Universidade Federal do Amazonas, Brasil \\ E-mail: erocha@ufam.edu.br \\ Rizioléia Marina Pinheiro Pina \\ ORCID: https://orcid.org/0000-0002-6114-4003 \\ Universidade Federal do Amazonas, Brasil \\ E-mail: rizioleia@ufam.edu.br \\ Douglas Fabiam de Oliveira Pôrto \\ ORCID: https://orcid.org/0000-0001-8019-4471 \\ Secretaria Municipal de Saúde de Manaus, Brasil \\ E-mail: Douglasfabiamdeoliveiraporto@gmail.com \\ Aimée de Queiroz Carvalho \\ ORCID: https://orcid.org/0000-0001-6901-8629 \\ Universidade Federal do Amazonas, Brasil \\ E-mail: aimee.queiroz@hotmail.com \\ Flávia da Silva Parente \\ ORCID: https://orcid.org/0000-0003-4947-0188 \\ Universidade Federal do Amazonas, Brasil \\ flaviaparenteoficial@gmail.com
}

\begin{abstract}
Resumo
Objetivo: Sistematizar a experiência vivenciada por acadêmicos de Enfermagem no telemonitoramento de pacientes suspeitos e/ou confirmados de COVID-19, por meio da ação estratégica "O Brasil Conta Comigo" na atenção básica em Manaus. Métodos: Trata-se de um estudo de natureza qualitativa, descritiva norteado pela metodologia de sistematização da experiência proposto por Holliday, utilizando-se, para tanto, de registros de observação e anotações, contatos com outros profissionais de saúde, estratégia de educação em saúde com usuários do SUS, aprendizado coletivo de novos saberes e práticas no enfrentamento da COVID --19, realizada no período de maio a dezembro/2020 no setor de telessaude da Secretaria Municipal de Saúde de Manaus, oportunizado por uma ação estratégica, conhecida como "O Brasil Conta Comigo" do Ministério da Saúde. Resultados: A vivenciada mostrou que o telemonitoramento, no cenário da pandemia, auxiliou na divulgação de informações e soluções de problemas sem necessidade de deslocamento aos serviços hospitalares. Instigou a busca por novos saberes sobre ferramentas tecnológicas de ensino e aprendizagem, bem como o uso da telessaúde, como alternativa, eficaz que facilita a comunicação com o paciente sem o contato físico e propicia um ambiente confortável e seguro para atenção digna à saúde, cumprindo assim, os princípios do SUS. Conclusão: O serviço de telemonitoramento funcionou de maneira eficaz durante o isolamento social servindo como estratégia de formação acadêmica, além de servir como alternativa à população que enfrenta fragilidades relacionadas aos aspectos sócioeconômicos e de acesso ao serviço de saúde.
\end{abstract}

Palavras-chave: Telemonitoramento; Infecção por coronavírus; Sistema Único de Saúde.

\footnotetext{
Abstract

Objective: Systematize the experience experienced by nursing students in the telemonitoring of patients suspected and/or confirmed of COVID-19, through the strategic action "Brazil Counts With Me" in primary care in Manaus. Methods: This is a qualitative, descriptive study based on the methodology of systematization of the experience proposed by Holliday, using, for this, observation records and notes, contacts with other health professionals, health
} 
education strategy with SUS users, collective learning of new knowledge and practices in coping with COVID --19, held from May to December/2020 in the telesaude sector of the Manaus Municipal Health Department, opportunistic by a strategic action, known as "Brazil Counts With Me" of the Ministry of Health. Results: The experience showed that telemonitoring, in the pandemic scenario, helped in the dissemination of information and problem solutions without the need to travel to hospital services. It instigated the search for new knowledge about technological tools of teaching and learning, as well as the use of technical health, as an effective alternative that facilitates communication with the patient without physical contact and provides a comfortable and safe environment for dignified health care, thus fulfilling the principles of the SUS. Conclusion: The telemonitoring service worked effectively during social isolation, serving as an academic training strategy, besides serving as an alternative to the population that faces weaknesses related to socio-economic aspects and access to health services.

Keywords: Telemonitoring; Coronavirus infection; Unified Health System.

\section{Resumen}

Objetivo: Sistematizar la experiencia experimentada por los estudiantes de enfermería en la telemonitorización de pacientes sospechosos y/o confirmados de COVID-19, a través de la acción estratégica "Brasil cuenta conmigo" en atención primaria en Manaos. Métodos: Este es un estudio cualitativo y descriptivo basado en la metodología de sistematización de la experiencia propuesta por Holliday, utilizando, para ello, registros de observación y notas, contactos con otros profesionales de la salud, estrategia de educación sanitaria con usuarios de SUS, aprendizaje colectivo de nuevos conocimientos y prácticas para hacer frente a COVID --19, celebrado de mayo a diciembre/2020 en el sector de telesaude del Departamento De Salud Municipal de Manaos, oportunista por una acción estratégica, conocida como "Brasil cuenta conmigo" del Ministerio de Salud. Resultados: La experiencia demostró que la telemonitorización, en el escenario de la pandemia, ayudó a la difusión de información y soluciones problemáticas sin necesidad de viajar a los servicios hospitalarios. Instigó la búsqueda de nuevos conocimientos sobre herramientas tecnológicas de enseñanza y aprendizaje, así como el uso de la salud técnica, como una alternativa eficaz que facilita la comunicación con el paciente sin contacto físico y proporciona un entorno cómodo y seguro para la atención sanitaria digna, cumpliendo así los principios del SUS. Conclusión: El servicio de telemonitorización funcionó eficazmente durante el aislamiento social, sirviendo como una estrategia de formación académica, además de servir como una alternativa a la población que se enfrenta a debilidades relacionadas con los aspectos socioeconómicos y el acceso a los servicios de salud.

Palabras clave: Telemonitorización; Infección por coronavírus; Sistema Unificado de Salud.

\section{Introdução}

No atual cenário de saúde mundial, a COVID-19 é um desafio para a saúde pública, pois a transmissibilidade do vírus ocorre por meio de secreções oriundas do sistema respiratório, favorecendo a transmissão através do toque, gotículas de saliva, espirro, tosse, objetos e superfícies contaminados, por esse motivo, percebe-se a facilitação da propagação viral (Junior et al., 2020).

Mesmo com as limitações relacionadas ao conhecimento acerca da doença, o Ministério da Saúde classifica a COVID19, como síndrome gripal, definindo como quadro respiratório agudo, tendo como manifestações mais comuns sinais de febre ou sensação febril, podendo ser acompanhada de tosse, dor de garganta, coriza, ou dificuldade respiratória, uma vez presente a dispneia, pressão torácica ou saturação de oxigênio inferior a 95\% em ar ambiente ou cianose nos lábios e/ou rosto, o quadro gripal passa a ser considerada uma síndrome respiratória aguda grave (Iser et al., 2020).

A apresentação clínica da COVID-19, pode variar de acordo com cada organismo, podendo apresentar-se de maneira assintomática, leve, moderada e grave. O período de incubação do vírus varia de um a catorze dias, com aparecimentos dos sintomas em torno de cinco a seis dias, tais como febre, fadiga, tosse seca, dificuldade de respirar, dispneia, edema de tonsilas, secreção mucóide, perda ou alteração do olfato e paladar, quadros diarréicos, náuseas, cefaléia. Quando confirmado o diagnóstico de COVID-19, porém sem presença de sintomas, classifica-se como assintomática, significando que há infecção pelo vírus, mas sem desenvolvimento de sinais e sintomas (Campos et al., 2020).

A estratificação de gravidade em indivíduos suspeitos de síndrome gripal é realizada considerando o quadro clínico do indivíduo, classificada em casos leves, na ausência de dispneia ou sinais e sintomas que indicam gravidade, nesse caso, podendo ser acompanhada pela Atenção Primária à Saúde (APS), quando a síndrome gripal é acompanhada por dificuldade respiratória, 
em indivíduos que não apresentam comorbidades, é necessário a estabilização na APS e encaminhamento ao centro de referência, serviços de pronto atendimento, urgência e emergência e/ou unidades hospitalares, em um período de vinte e quatro horas, por exigir um suporte tecnológico em exames de imagem e laboratoriais que auxiliam no diagnóstico e conduta clínica pela equipe de saúde (Iser et al., 2020).

A proliferação acelerada da doença impulsionou medidas como quarentena nas comunidades, uma intervenção de Saúde Pública, orientada pela OMS, para reduzir os casos de COVID-19, desse modo, foi sugerido em diversos países, incluindo o Brasil, o isolamento social, como estratégia para desenfrear essa cadeia de transmissão (Szwarcwald, 2020).

Com a quarentena, foi introduzido o lockdown, uma medida radical com a proibição de eventos públicos, funcionamento de escolas, ambientes turísticos, "shoppings" e quaisquer ambientes que propiciassem aglomerações (Costa et al., 2020). As medidas adotadas tinham o intuito de favorecer o distanciamento social, concomitante a essa medida, também foi recomendado à utilização de máscaras, adequada higienização das mãos, utilização de álcool em gel, visando a proteção individual e redução da disseminação da doença por infecção cruzada (Oliveira et al., 2020).

No intuito de aumentar o contingente de pessoal e otimizar a oferta de serviços no âmbito do Sistema Único de Saúde (SUS), o Ministério da Saúde (MS) instituiu a estratégia "Brasil Conta Comigo" através da portaria de n 492 , de 23 de março de 2020, direcionada a alunos dos cursos da área de saúde que ao se inscreverem se voluntariavam a atuar enquanto perdurasse a pandemia, tal portaria específica seu direcionamento aos cursos de enfermagem, medicina, farmácia e fisioterapia. A adesão de Estratégia ficou a critério de Estados, Municípios e Distrito Federal através da formalização dos gestores locais de SUS, via sistema eletrônico (Brasil, 2020).

Em virtude do avanço da pandemia, um dos serviços que ganhou destaque foi a telessaúde, pois proporcionava a teleconsulta por meio de chamadas de áudio e/ou vídeo, recurso que reduz a circulação de indivíduos nos serviços de saúde, diminuindo os riscos de transmissão do vírus (Caetano et al., 2020).

Em Manaus, a Secretaria Municipal de Saúde (SEMSA) implantou o serviço de Telemonitoramento no âmbito da Central de Regulação Regional de Manaus, no qual os voluntários da Estratégia "Brasil Conta Comigo" eram inseridos por meio de um supervisor para auxiliar os demais profissionais da área de saúde que estavam envolvidos na telessaúde no âmbito da atenção básica.

Portanto, o objetivo desse manuscrito é sistematizar a experiência vivenciada por acadêmicos de Enfermagem no telemonitoramento de pacientes suspeitos e/ou confirmados de COVID-19, por meio da ação estratégica “O Brasil Conta Comigo" na atenção básica do município de Manaus.

\section{Metodologia}

O relato de experiência é uma ferramenta que possibilita a descrição da vivência em determinado período e circunstância, podendo utilizar o método qualitativo, que para Pereira (2018), contribui para análise interpretativa da atividade desenvolvida, pois possibilita ao pesquisador, analisar a representatividade que o indivíduo dar a determinada situação ou objeto.

Portanto, trata-se de um estudo de natureza qualitativa, descritiva do tipo relato de experiência focada na vivência no setor de telessaúde, no âmbito da Secretaria Municipal de Saúde de Manaus (SEMSA), oportunizado por meio da Estratégia Brasil Conta Comigo, no período de maio a dezembro de 2020, com a duração de quarenta horas semanais.

A participação nessa estratégia ocorria através da inscrição do aluno no site Ministério da Saúde e realização do curso online e gratuito "Protocolos de manejo clínico para enfrentamento ao covid-19”, após a inscrição e realização do curso o aluno aguardava a convocação, onde constaria o local a ser desenvolvida a ação e o seu supervisor (Brasil, 2020).

O manuscrito relata a vivência de acadêmicos do $9^{\circ}$ período do curso de graduação em Enfermagem da Universidade Federal do Amazonas durante a participação no serviço de telessaúde, por meio do telemonitoramento de pacientes com casos 
suspeitos ou notificados com COVID-19 no município de Manaus. Esse telemonitoramento é uma iniciativa da SEMSA, dentro do telessaúde, que faz parte da Central de Regulação Regional de Manaus, como uma forma de reduzir os casos de COVID-19 na capital do Amazonas, assim como interceptar a cadeia de transmissão (Manaus, 2020).

O serviço era constituído por uma equipe multidisciplinar composta por enfermeiros, médicos, farmacêuticos, assistentes sociais, psicólogos e odontólogos, dentre esses havia aqueles com suas respectivas especializações.

Posteriormente, com a Portaria de n492 de 23 de março de 2020, a SEMSA, introduziu acadêmicos da área da saúde no âmbito do SUS em alguns de seus serviços. No setor de telessaúde, foram inseridos discentes finalistas dos cursos de medicina e enfermagem, para atuarem como operadores, juntamente com a equipe de profissionais, para tanto, foram a realizadas atividades de educação continuada, com vistas a promoção de um mesmo entendimento e mesma linguagem entre os profissionais e acadêmicos de saúde, no momento do atendimento ao usuário do SUS.

A educação continuada era realizada pelos próprios profissionais do telessaúde ou profissionais convidados que possuíssem expertise na temática abordada. As atividades eram comumente realizadas em uma sala separada, local onde aconteciam as aulas por transmissão de vídeo chamada para os demais profissionais e acadêmicos, evitando, aglomeração.

\section{Resultados}

\section{A. O ponto de partida: viver a experiência.}

A pandemia coincidiu com o início das aulas do curso de graduação em enfermagem, da Universidade Federal do amazonas, nesse período os discentes já haviam completado $75 \%$ da carga horária obrigatória total do curso, o que favoreceu um arcabouço teórico e prático com significativa contribuição para a formação profissional.

Contudo a pandemia alterou a programação curricular e frente à necessidade de distanciamento social e da interrupção das aulas presenciais, a continuidade do estágio em Enfermagem foi garantida por meio da medida provisória $\mathrm{n}^{\circ}$ 934, que assegurou o término do curso ao estagiário durante o período da pandemia. A medida prevê normas de caráter excepcional para o ensino superior durante o enfrentamento da situação de saúde pública atual.

Nesse cenário destacou-se a importância do estudante de enfermagem, que ganhou visibilidade em âmbito nacional, evidenciado pela da portaria 492, de 23 de março de 2020, da estratégia Brasil Conta Comigo, que visava contemplar alunos dos cursos de enfermagem, medicina, farmácia e fisioterapia, convocando para atuar em diversos setores da área da saúde, tendo apresentado a carga horária de vinte horas semanais para discentes dos períodos iniciais e quarentas horas semanais para acadêmicos finalistas, proporcionando a experiência durante a pandemia.

A inscrição na estratégia partia da iniciativa dos discentes e autorização da universidade, os alunos finalistas foram estimulados à participarem, com o incentivo do edital, após confirmação da inscrição a discente foi convocada para atuar na Central de Regulação Regional de Manaus, dentro do setor de telessaúde que atua também com o telemonitoramento de pacientes suspeitos ou confirmados com COVID-19, juntamente com o direcionamento do profissional responsável pela supervisão do acadêmico convocado.

\section{B. As perguntas iniciais.}

As perguntas iniciais estão norteadas pela participação na Estratégia Brasil Conta Comigo frente à pandemia do novo coronavírus. Desse modo as perguntas realizadas nesse estudo são: “Quais estratégias de saúde poderiam ser adotadas pelo acadêmico de enfermagem durante a pandemia?” e “Qual a relevância da participação na Estratégia Brasil Conta Comigo para a formação em enfermagem?". 


\section{A recuperação do processo vivido.}

Com a finalidade de alinhar o discurso da equipe, foram realizadas atividades de educação continuada, com aulas sobre a temática COVID-19, fisiopatologia do novo coronavírus, sinais e sintomas mais comuns e orientações necessárias para casos suspeitos e confirmados de COVID-19.

Após as atividades de educação continuada foram realizados treinamentos acerca dos procedimentos e condutas para gestantes e puérperas acometidas pela covid-19, havia a preocupação acerca de manter a equipe alinhada nas informações para que todos tivessem o mesmo direcionamento, para que as informações fossem adequadas e baseadas nas melhores evidências científicas contribuindo para uma gestação e puerpério o mais saudável, mesmo com as intercorrências da COVID-19, além de treinamentos que relacionavam a Covid-19 com senescência e comorbidades frequentes nesse período, além de ênfase em abordagem na pediatria e dermatologia.

As ações desenvolvidas eram realizadas por meio de ligações para pacientes suspeitos e confirmado de COVID-19, sintomáticos ou assintomáticos, que aguardavam o teste ou ao fim dos catorze dias de isolamento e que foram notificados no município de Manaus, em parceria com o Centro de Informações em Vigilância em Saúde - CIEVS, Secretária de Vigilância em Saúde, Fundação de Vigilância em Saúde - FVS e Secretaria Municipal de Saúde de Manaus - SEMSA.

Por meio de um prontuário eletrônico interno, era feita a classificação de acordo com a sintomatologia relatada, podendo ser assintomático, leve, moderado ou grave, conforme a melhora ou agravamento do paciente. Durante o atendimento eram passadas as orientações adequadas à situação de saúde.

As informações coletadas além de serem inseridas no prontuário interno, eram também inseridas no Prontuário Eletrônico do Cidadão - PEC pelos profissionais registrados em conselhos, e em Sistema de Coleta de Dados Simplificada CDS pelos acadêmicos, sob observação do supervisor, direcionado pela Estratégia Brasil Conta Comigo.

\section{Reflexão de fundo.}

Durante o período de vivência no telemonitoramento de pacientes suspeitos ou confirmados com COVID-19, foram necessárias constantes atualizações, por se tratar de uma doença nova e com diversos estudos surgindo em torno do Sars-CoV2. O cenário da pandemia instigou a busca por novos saberes e a desconstrução de uma percepção em torno do telessaúde, facilitando a comunicação com o paciente sem o contato físico e propiciando um ambiente confortável e seguro para acompanhamento do usuário de saúde infectado.

A experiência favoreceu conhecer diversas histórias por trás dos números atualizados pela vigilância epidemiológica do Amazonas, histórias muitas vezes acompanhadas pela perda de um ente querido para a COVID-19, e histórias de superação.

Durante o atendimento era perceptível a falta de informação ou informações equivocadas baseadas em especulações populares, com isso, era feito a intervenção, buscando esclarecer as dúvidas concernentes ao COVID-19 e orientação quanto ao isolamento social e medidas de autocuidado e prevenção.

Passar pelo processo, favoreceu o entendimento da importância de atuar em uma equipe multiprofissional, conhecer a função e importância de cada uma das profissões na área da saúde para recuperação e bem estar do paciente.

A experiência possibilitou o alcance de lugares longínquos e de difícil acesso, através da telessaúde, por meio de plataformas virtuais, como ferramenta para educação em saúde, sendo vista como um campo de atuação da enfermagem, que é ensinado durante toda a graduação, possibilitando o desenvolvimento de habilidades e competências para agir com segurança.

\section{E - O ponto de chegada.}

A experiência vivida durante o período de março a outubro de 2020, gerou reflexões capazes de responder as perguntas descritas nas segundas etapas, além de trazer contribuições para a formação em enfermagem. 
A experiência favoreceu, o reconhecimento da diferença entre as práticas realizadas no âmbito da graduação, que os acadêmicos estavam habituados, com o atual cenário encontrado nos serviços de saúde durante a pandemia, dessa forma, houve a necessidade de readaptação, favorecida pela participação no serviço de telemonitoramento através da estratégia Brasil Conta Comigo, que ampliou o olhar concernente a situação atual vivenciada, colaborando para o desenvolvimento de competências e habilidades para a formação do profissional enfermeiro.

As situações vividas no atual cenário colaboraram para a reflexão acerca das necessidades de estratégias de readequação para exercer a profissão de enfermagem, tais como a utilização de ferramentas virtuais como estratégia para educação em saúde, com vistas a esclarecimento de dúvidas e orientações, baseadas nas melhores evidências científicas para promoção da saúde e prevenção de doenças e/ou agravos.

A participação na Estratégia Brasil Conta Comigo, acrescentou na prática de enfermagem e no desenvolvimento profissional, gerando segurança e suporte teórico e prático para o exercício da profissão, por propiciar um ambiente seguro, sendo possível realizar uma escuta qualificada e vivenciar na prática todos os conhecimentos adquiridos durante a graduação, dentro de um contexto pandêmico.

A participação na estratégia contribuiu para a formação em enfermagem, por favorecer a capacitação teórica antes de adentrar ao serviço de saúde, possibilitando ambiente favorável a discussões de casos clínicos e desenvolvimento de pensamento crítico, fundamental para o exercício da profissão, ancorados em fundamento técnicos-científicos com capacidade de impulsionar na melhora da qualidade da assistência à saúde.

\section{Considerações Finais}

O cenário pandêmico trouxe mudanças no mundo, em todos os níveis de atenção à saúde. Atuar na estratégia Brasil Conta Comigo, no setor de telessaúde, oportunizou o compartilhamento de conhecimentos, agregou novos aprendizados, e possibilitou a execução na prática de todo conhecimento adquirido durante o curso de graduação em Enfermagem.

Fazer telemonitoramento por meio da telessaaúde de pacientes com suspeita ou infectados pela COVID-19, permitiu o exercício da função do enfermeiro como educador em saúde, mesmo à distância. Por meio das sistematizações foram produzidos documentos que servem de instrumento para as ações das equipes de saúde técnicas.

A permanência dos estudantes de enfermagem junto aos serviços de saúde no combate ao COVID-19 produziu importantes experiências e vivências na integralidade do cuidado, na intervenção e prevenção durante a pandemia frente aos fatores de risco, bem como na promoção da saúde da comunidade.

Por fim, enfatizamos que as circunstâncias vivenciadas em um cenário pandêmico, favoreceram a readequação de estratégias por meio da teleconsulta, desenvolvimento de resiliência frente às situações adversas enfrentadas, com foco na resolutividade dos problemas identificados e vistas a propiciar a oferta de serviços de saúde de qualidade à comunidade.

\section{Referências}

Brasil (2020). Ministério da Saúde. Gabinete do Ministro. Portaria nº 492, de 23 de março de 2020.

Caetano, R., Silva A. B., \& Carneiro, A. C. (2020) Desafios e oportunidades para telessaúde em tempos da pandemia pela COVID-19: uma reflexão sobre os espaços e iniciativas no contexto brasileiro. Cadernos de Saúde Pública. 36(5).

Costa, M. F. L. (2020). Distanciamento social, uso de máscaras e higienização das mãos entre participantes do Estudo Longitudinal da Saúde dos Idosos Brasileiros: iniciativa ELSI-COVID-19. Cadernos de Saúde Pública [online], 36(3). https://doi.org/10.1590/0102-311X00193920.

Oliveira, A. C. (2018). O que a pandemia da covid-19 tem nos ensinado sobre adoção de medidas de precaução? Texto contexto - enferm: Florianópolis. https://doi.org/10.1590/1980-265x-tce-2020-0106.

Fernandes, F. D. M. (2020). Uso de telessaúde por alunos de graduação em Fonoaudiologia: possibilidades e perspectivas em tempos de pandemia por COVID19. CoDAS, 32(4). https://doi.org/10.1590/2317-1782/20192020190. 
Research, Society and Development, v. 10, n. 2, e39910210857, 2021

(CC BY 4.0) | ISSN 2525-3409 | DOI: http://dx.doi.org/10.33448/rsd-v10i2.10857

Holliday, O. J (2006). Para sistematizar experiências/Oscar JaraHolliday; tradução de: Maria Viviana V. Resende. 2. ed., revista. Brasília: MMA. Disponível em: https://www.mma.gov.br/estruturas/168/_publicacao/168_publicacao30012009115508.pdf.

Iser, B. P. M. (2020). Definição de caso suspeito da COVID-19: uma revisão narrativa dos sinais e sintomas mais frequentes entre os casos confirmados. Epidemiol. Serv. Saúde [online]. 29(3), e2020233. http://dx.doi.org/10.5123/s1679-49742020000300018.

Manaus, Prefeitura Municipal de. (2020). Secretaria Municipal de Finanças, Planejamento e Tecnologia da Informação - SEMEF. Monitoramento dos casos de COVID-19: Manaus. Encontrado em: https://covid19.manaus.am.gov.br/monitoramento/.

Pereira A. S. (2018). Metodologia da pesquisa científica. UFSM. https://repositorio.ufsm.br/bitstream/handle/1/15824/Lic_Computacao_Metodologia-PesquisaCientifica.pdf?sequence $=1$

Souza J. R., Cruz, R. C. R., Brito V. C., \& Santos E. L. S. (2020). COVID-19 e a promoção da saúde em tempos de pandemia. Revista eletrônica acervo saúde. https://doi.org/10.25248/reas.e3837.2020

Schramm, J. M. A., \& Emmerick, I. C. M. (2020) Carga de doença da COVID-19 e de suas complicações agudas e crônicas: reflexões sobre a mensuração (DALY) e perspectivas no Sistema Único de Saúde. Cad. Saúde Pública. 36(11). https://doi.org/10.1590/0102-311x00148920.

Szwarcwald, C. L. (2020). Adesão às medidas de restrição de contato físico e disseminação da COVID-19 no Brasil. Epidemiologia e Serviços de Saúde. 29(5). https://doi.org/10.1590/S1679-49742020000500018. 